\title{
Conformationally Constrained Ac-His-D-Nal(2')-Nle-Trp-NH2 Analogues Leads to Selective Melanotropins
}

\author{
Saghar Mowlazadeh Haghighi, Minying Cai*, Yang Zhou, Jonathon Sawyer, \\ Bailey Lahtinen, Charley Chen, and Victor Hruby \\ Department of Chemistry, University of Arizona, Tucson, AZ, 85721, USA
}

\section{Introduction}

Skin pigmentation shows substantial variation within human populations and within regional groupings or races. High levels of UVR can lead to skin damage such as sunburn or skin cancer, as well as the breakdown of folic acid. Melanin provides some protection from UVR induced damage [1]. MC1-R has a well-defined role in the regulation of skin pigmentation, provides protection against damaging and mutagenic effects of UV radiation (skin cancer) and has been recently implicated in the control of inflammation [2,3].

\section{Results and Discussion}

Introduction of stereochemical modifications (D-amino acids), functional group modification, conformational constraints, and topographical constraints to the "core" peptide sequence provide an effective means to investigate these preferences [4]. Aromatic amino acids (Phe, Tyr, Trp, and His) are often a key element in bioactive peptides [5]. The procedure allows the introduction of a variety of substituents (halogens) at the phenylalanine derivatives. Phenylalanine and histidine are also conserved among all five melanocortin receptors. Mutation of His to proline resulted in significant decrease in endogenous agonist binding and receptor signaling, suggesting that these residues are also involved in melanocortin orthosteric binding. Therefore, in our effort to produce selective melanocortin ligands, we devoted a great deal of time to obtaining ligands with selective activity for the hMC1R and to developing the pharmacological properties that are suitable for medical applications of these compounds. Among our synthesized peptides, Ac-His-4-Br-D-Phe-Nle-Trp-NH 2 (1), Ac-His4-Cl-D-Phe-Nle-Trp- $\mathrm{NH}_{2}$ (2), show enhanced drug efficacy and MC1R activity compared to our previously synthesized tetrapeptides.

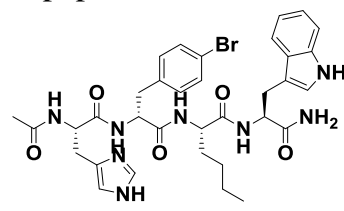

(1)

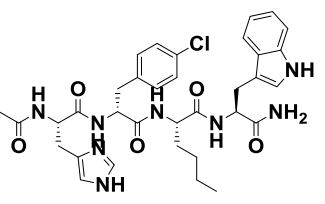

(2)

Fig. 1. Novel selective peptides.

Peptide Design and Synthesis. We have used standard solid-phase methods. The peptides were synthesized on Rink Amide resin using Fmoc chemistry. Initially, the resin was deprotected with $20 \%$ (v/v) piperidine in DMF solution. The first amino acid was coupled to the resin. The following amino acids were coupled sequentially to the growing peptide chain. Each coupling reaction was achieved using of amino acid, HCTU in the presence of DIEA. The peptide was cleaved from the resin, and the other side chain protecting groups were removed in a cleavage cocktail with $95 \%$ TFA, $2.5 \%$ TIS, $2.5 \%$ water.

In conclusions, one of the major goals of medicinal chemistry has been the design and synthesis of novel peptides with conformationally constrained side chains in order to obtain highly selective and potent peptide analogues. Our biological data suggests that these compounds are selective MC1R agonists and have the $\mathrm{EC}_{50}$ within nanomolar range. Substitution of Nle5 produced a more balanced activity in these peptides. 
Table 1. Binding and cAMP Assays of Novel Peptides toward Melanocortin Receptors.

\begin{tabular}{|c|c|c|c|c|c|c|c|c|c|c|c|c|c|c|c|c|}
\hline & \multicolumn{4}{|c|}{$M C 1 R$} & \multicolumn{4}{|c|}{$M C 3 R$} & \multicolumn{4}{|c|}{$M C 4 R$} & \multicolumn{4}{|c|}{$M C 5 R$} \\
\hline & \multicolumn{2}{|c|}{ Binding } & \multicolumn{2}{|c|}{$c A M P$} & \multicolumn{2}{|c|}{ Binding } & \multicolumn{2}{|c|}{$c A M P$} & \multicolumn{2}{|c|}{ Binding } & \multicolumn{2}{|c|}{$c A M P$} & \multicolumn{2}{|c|}{ Binding } & \multicolumn{2}{|c|}{$c A M P$} \\
\hline & $\mathrm{IC}_{50}$ & $\% \mathrm{BE}$ & $\mathrm{EC}_{50}$ & Act $\%$ & $\mathrm{IC}_{50}$ & $\% \mathrm{BE}$ & $\mathrm{EC}_{50}$ & Act $\%$ & $\mathrm{IC}_{50}$ & $\% \mathrm{BE}$ & $\mathrm{EC}_{50}$ & Act $\%$ & $\mathrm{IC}_{50}$ & $\% \mathrm{BE}$ & $\mathrm{EC}_{50}$ & Act $\%$ \\
\hline (1) & 1621 & 61 & 9.6 & 100 & NB & 0 & 111 & 93 & 189 & 46 & 40 & 79 & NB & 0 & NA & 0 \\
\hline (2) & 1053 & 81 & 8.5 & 100 & $>10000$ & 45 & 102 & 87 & NB & 0 & 19 & 55 & NB & 0 & NA & 0 \\
\hline MT-II & $1 \pm 0.1$ & 100 & $1.02 \pm 0.4$ & 100 & $2.0 \pm 0.1$ & 100 & $5.1 \pm 0.3$ & 100 & $2.3 \pm 0.85$ & 100 & $2.1 \pm 0.6$ & 100 & $4.2 \pm 1.3$ & 100 & $5.7 \pm 2.2$ & 100 \\
\hline
\end{tabular}

${ }^{a} M T-I I=A c-N l e-c[A s p-H i s-D-P h e-A r g-T r p-L y s]-N H 2 .{ }^{b} I_{50}=$ concentration of peptide at $50 \%$ specific binding $(N=4) . N B=0 \%$ of $125 I-N D P-\alpha-M S H$ displacement observed at $10 \mu \mathrm{M} . \% B E$ (binding efficiency) = maximal $\%$ of 125I-NDP- $\alpha$-MSH displacement observed at $10 \mu \mathrm{M}$. EC $C_{50}=$ Effective concentration of peptide that was able to generate $50 \%$ maximal intracellular cAMP accumulation $(N=4)$. Act $\%=\%$ of cAMP produced at $10 \mu M$ ligand concentration, in relation to MT-II. NA $=0 \%$ cAMP accumulation observed at $10 \mu M$. The peptides were tested at a range of concentration from $10^{-10}$ to $10^{-5} \mathrm{M}$.
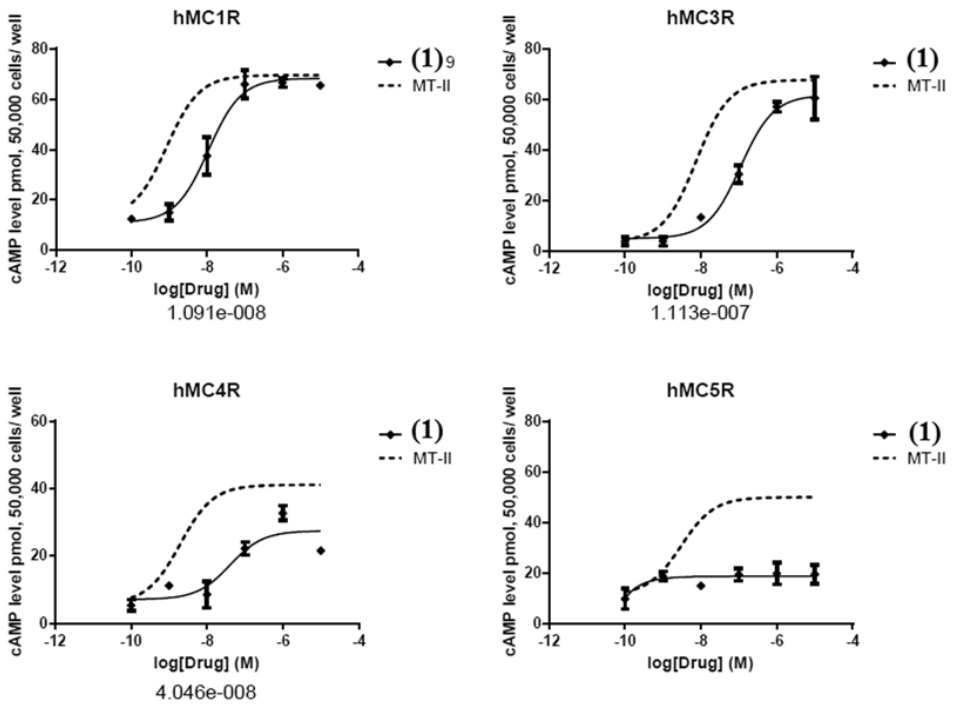

Fig. 2. The comparison between Melanocortin activity of synthesized Peptides and MT-II.

\section{Acknowledgments}

Supported in part by a grant from the U.S. Public Health Service, National Institutes of Health, DK017420, GM 108040 and DA06284.

\section{References}

1. Norton, H.L., Friedlaender, J.S., Merriwether, D.A., Koki, G., Mgone, C.S., Shriver, M.D. Am. J. Physical Anthropology 130, 254-268 (2006), http://dx.doi.org/10.1002/ajpa.20343

2. Rinne, P., Ahola-Olli, A., Nuutinen, S., Koskinen, E., Kaipio, K., Eerola, K., Juonala, M., Kähönen, M., Lehtimäki, T., Raitakari, O.T., Savontaus, E. Arterioscler. Thromb. Vasc. Biol. 35, 1678-1686 (2015), http://dx.doi.org/10.1161/ATVBAHA.114.305064

3. Ying, J., Gu, X., Cai, M., Dedek, M., Vagner, J., Trivedi, D.B., Hruby, V.J. J. Med. Chem. 49, 6888-6896 (2006), http://dx.doi.org/10.1021/jm060768f

4. Morgan, A.A., Rubenstein, E. PLoS One 8, 53785 (2013), http://dx.doi.org/10.1371/journal.pone.0053785

5. Pulka, K., et al. Tetrahedron 63, 1459-1466 (2007), http://dx.doi.org/10.1016/j.tet.2006.11.069 